\title{
Optimized Designing of Optical Local Area Network With Optical Code Division Multiple Access Incorporating Forward Error Correction
}

\author{
Aqeel Hamzah Sahib AL-fatlawi, A.K.Jaiswal, Mukesh Kumar \\ M.tech Student ,Department of Electronics and Communication Engineering \\ Sam higginbottom Institute of Agriculture, Technology and Science, Allahabad, Uttar Pradesh,India-
} 211007

\begin{abstract}
Optical Code-Division Multiple-Access (OCDMA) have flourished as successful schemes for expanding the transmission capacity as well as enhancing the security for OCDMA. Optical code-division multiple-access is proposed as a natural solution to achieving asynchronous, high-speed connectivity in a local area network environment. Optical CDMA is shown to be competitive with other networking technologies such as WDMA and TDMA, but has the benefit of more flexibility, simpler protocols, and no need for centralized network control. The purpose of this paper is to review the literature on OCDMA overlay systems, including our approach of one dimensional coding of SAC OCDMA. Furthermore, an essential background of OCDMA, recent coding techniques and security issues are also presented.The limitations of one-dimensional optical orthogonal codes for CDMA have motivated the idea of spectral spreading in both the temporal and wavelength domains. If the constraints on constant weight in these two-dimensional codes are relaxed, differentiated levels of service at the physical layer become possible. Areas for further research are suggested which may allow quality of service levels to be guaranteed at the physical layer.
\end{abstract}

Keywords: OCDMA, multiple access, OOC(Optical orthogonal code), WDMA+CDMA, Multiple weight OOC,

\section{Introduction}

Emerging of wireless CDMA telephony in the 1980swith tremendous benefits of CDMA such as a merit of Spread Spectrum (SS) pushes some researchers to transfer principles and advantages of CDMA into optical communication networks $[1,2,3]$.The first experiment that demonstrates the feasibility of implementing CDMA thought into the optical domain was in 1986 by [4]. Then Salehi employed CDMA techniques in optical systems and analyzed their performances [5,6].Up till now, interest in applications of OCDMA has been constantly rising during recent decades [7].The nature of OCDMA in security has been concerned for some application of enterprise and military networks. To increase the security degree, the hybrid system of both OCDMA and WDM has been studied, in addition to improve the capacity of simultaneous users. This hybrid scheme is classified into: (1) OCDMA channels-over-WDM, whereby WDM is employed as a medium, (2) Inband transmission of both OCDMA and WDM signals. Most previous reviews in this field focus on pure OCDMA systems and optical coding through their applications. In contrast to the earlier work, this paper reports an essential review of OCDMA systems and its related security. In addition, we survey the current trends in hybrid OCDMA and WDM schemes, including the type of OCDMA coding, security enhancement as well as the systems performance. Typical LAN traffic is bursty by nature. Therefore, static channelization (or multiplexing) schemes, realized by permanently allocated time- or wavelength division multiplexing (TDM, WDM)slots waste resources for idle users [8]. Users can share the communication bandwidth more effectively by using dynamic medium access techniques (eg statistical multiplexing). This can be realized for instance by code division, or carrier sense multiple access techniques(CDMA, CSMA).

Let us now inspect the realization of CDMA by employing spreading and dispreading in optical domain. In intensity modulation, direct detection (IM-DD) non-coherent optical communication systems, intensity of the optical signal is modulated and detected. Therefore, only unipolar codes can be applied for optical, non-coherent multiple access. Optical Orthogonal Codes (OOC) [9] are proposed for non-coherent optical CDMA. The OOC scan be generated simply by using optical passive components, such as optical delay lines, optical splitters and optical combiners [10]. This work extends the earlier results in [9] to the case of different weight and different rate users while preserving the original correlation properties of the OOCs. In section 2, the original OOC system is reviewed and the multi-weight, multi-length OOC is introduced. In order to illustrate our ideas, we describe an OOC based system with two user classes. In order to support multiple rate, and QoS differentiation, the construction of strict OOC can be generalized based on the concept of mark position difference set. The code set construction is flexible in such a way that the code length and weight can be selected arbitrarily as long as the number of desired codes in each class of users can be supported. 
1.1 Optical CDMA Background: Various multiple access techniques have been developed for optical fiber communications. This can provide a large bandwidth by exploitation of the optical medium capacity. Then, many channels are combined together over a single fiber link. There are three main ways to employ the multiplexing technique in optical networks. Time-Division Multiple Access (TDMA) scheme assigns users to transmit data over a dedicated time slot from a number of accessible slots. However, TDMA suffers from a hard limitation of the number of users that is based on the availability of this resource. The second technique is Wavelength-Division Multiple Access(WDMA). Systems that operate by WDM assign a unique wavelength from a number of available wavelengths. On the other hand, in OCDMA, each user has a unique code as an assignment address that spreads over a relatively wide bandwidth.

\subsection{Multiple-Weight Two-Dimensional Codes:}

In the late 1990s, Kwong and Yang developed a new class of MWOOCs without the restrictions of one constant code weight $[11,12]$. Through the algebraic code generation technique, two different classes of code words were created: low weight $w$ and high weight $2 w$. These multiple-weight MWOOCs are the key to providing different QoS levels in an optical CDMA network at the physical layer in the OSI model. The MWOOCs in general perform better than WDMA +CDMA schemes for a given bandwidth expansion and number of wavelengths. Designing 2D signature sequences, rather than artificially partitioning the available bandwidth into independent wavelength channels, leads to a lower probability of error. Various techniques have been developed to construct

MWOOCs such as modification of 1D OOCs, application of error correction codes to the optical domain, or algebraic coding methods. In all cases, however, the codes were arbitrarily restricted to have a single pulse per row in the matrix. This limited the code cardinality and necessitated equal weight for all signature sequences. As we show in the following section, relaxing the single pulse per row constraint will lead to a more flexible family of MWOOCs that may allow for differentiated levels of service at the physical layer in a CDMA LAN.

This specific code is modulated and then a message signal is transmitted at an arbitrary time to an intended receiver, which can match the correct code to recover the encoded information. The main OCDMA and WDM systems have been of wide spread implementation for local and metro access network[13,14]. This is because OCDMA systems provide users both simultaneous and asynchronous access to networks with high security $[15,6]$ and WDM systems supply a relatively high transmission capacity [16].

\subsection{Sharing the Fiber Optic Medium Using Codes}

\section{System Design Model}

We need to find a way to share the vast bandwidth of the optical fiber medium in a manner which is fair and fast. Three multiple access approaches are often considered to make the system bandwidth available to the individual user: time-division multiple accesses (TDMA), wavelength-division multiple access (WDMA), and code-division multiple access (CDMA).TDMA accommodates a large number of active users by interleaving bits from different sources into a period equal to that of the uncompressed bit. TDMA systems offer a large number of node addresses; however, the performance of TDMA systems is ultimately limited by the time-serial nature of the technology. The receiver must operate at the aggregate bit rate of the system, which is roughly equal to the number of nodes connected times the data rate per node. Even a modest system with only 100 users each communicating at $1 \mathrm{~Gb} / \mathrm{s}$ would result in a total throughput well beyond the typical electronic hardware speeds of several gigabits per second. TDMA systems also require strong centralized control to allocate time slots and maintain synchronous operation. Although centralized control is straight forward to implement in a LAN, the extra time required for users to request time slots and for the central node to allocate the slot may introduce significant latency. Furthermore, the allocation of dedicated time slots does not allow TDMA to take full advantage of statistical multiplexing gain, which may be significant when the data traffic is burst

In the WDMA approach, the available optical bandwidth is divided into distinct wavelength channels that are used concurrently by different users to achieve multiple access. If each wavelength channel were to support a data rate equal to the peak electronic capacity of several gigabits per second, a WDMA system with around 160 wavelength channels could accommodate on the order of a terabit per second of aggregate capacity.

\subsection{Fundamentals of OCDMA}

The general principle of coding and decoding of OCDMA is clarified. In coding operation, the spectrum of data signal is broadened as spread spectrum designation. Each user has been assigned to some chips of the code sequences to share the same transmission line using power splitters or combiners. This operation can be performed in the optical-domain and/or in the space-domain as well. Decoders at the receiver recognize a target code by employing match filtering. 


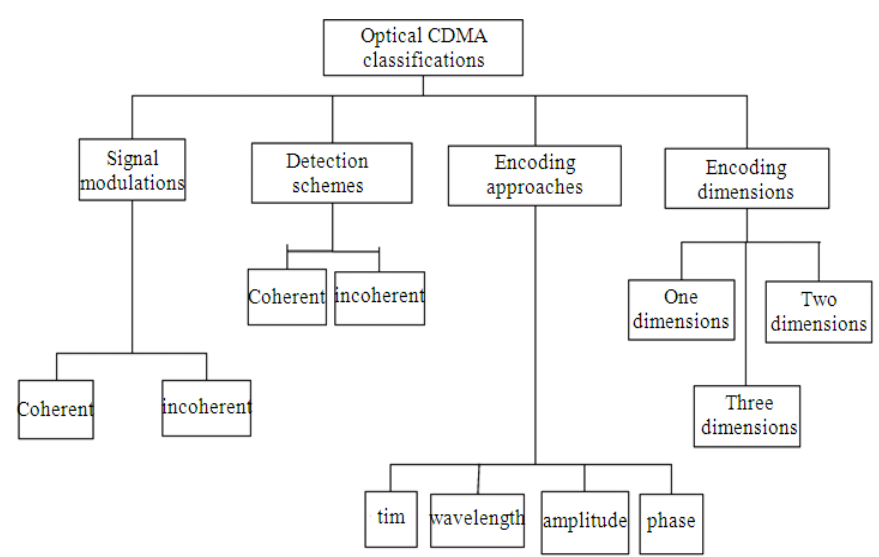

Fig. 1: Optical CDMA system classifications

It is important to produce high auto-correlations of desired codes, while undesired codes produce cross correlation with low-level power. Usually, cross correlations are presented between two different codes. An excellent code design has a relatively high code length with high auto-correlation for many users. Finally, electrical thresholding is performed to recover the original data. Figure 1 shows the summary of OCDMA classifications. Two categories of OCDMA that are classified in terms of signal modulation and detection schemes are coherent and incoherent systems. In a coherent OCDMA scheme, the signal modulation is based on optical phase coding which is often derived from a highly coherent source such as a Mode-Locked Laser (MLL) and detection at a receiver includes knowledge of the phase information of carriers.

If we categorize these systems based on coding optical signals approaches, the six types of coding are available as follows:

$>$ Direct-sequence or temporal coding optical CDMA systems

$>$ Spectral Amplitude Coding (SAC) Optical CDMA systems

$>$ Spectral Phase Coding (SPC) optical CDMA systems

$>$ Temporal phase coding optical CDMA systems;

$>$ Two-Dimensional (2-D) spatial or spread space coding optical CDMA systems

$>$ hybrid coding optical CDMA systems.

In the hybrid coding systems, combined coding techniques that mentioned above is used in one scheme. For example, Wavelength-Hopping Time-Spread (WHTS)encoding is 2-D coding scheme based on merging of spectrum encoding with temporal encoding Combination of the space encoding with previous 2-D coding, Space-Spread Wavelength-Hopping Time-Spreading encoding (SS-WH-TS) is generated and considered 3-D coding scheme.

\subsection{Optical CDMA Security}

Security over the optical communications network gives numerous challenges to both network providers and intruders and it experiences the same sensitivities and vulnerabilities as wireless communications. Optical CDMA is a kind of multiplexing for networks of optical communications in which optical pulses are processed by encoding/decoding data applying simple and low cost passive optical components. Its main advantages include asynchronous random access, supporting multiple rates, good compatibility with other multiplexing; TDM and WDM, flexible networking and providing some privacy of transmission information. OCDMA is a potential system that can boost the security for subscribers. This is because OCDMA encoded signals manifest itself as a noise-like waveform that possibly would not be available to an eavesdropper without knowing and understanding the assigned code of the authorized user. However, it's important for engineers to know the statement that is nothing of security for a single active user in OCDMA system that employs OOK modulation.

\subsection{WDM/OCDMA system}

In this scheme, codes of OCDMA can be employed on each WDM wavelength. Recently, this kind of hybrid system has been widely researched because it can support many more users with high data rates. Consequently, several new codes for OCDMA have been proposed and studied over the hybrid architecture. The WDM/SAC Balanced Incomplete Block Design (WS-BIBD) code was used to support a larger number of active users by reducing the effect of the PIIN noise. The double weight (DW) code family in a hybrid WDM/SAC system has also been studied. However, these systems are not capable of providing flexible and confidential access to optical networks. Maximal length sequence (M-sequence) codes were exploited by merit of the cyclic properties of Arrayed Waveguide Grating(AWG) routers. For more developed systems, the novel OCDMA 
encoders/decoders for different WDM channels have been proposed, where a phase mask was applied on a Super-Structure fiber-Bragg-Grating (S-SBG) based encoder/decoder to construct A Wavelength-DivisionMultiplexing (WDM) compatible OCDMA system. This led to good encoding efficiency and correlation performance.

\section{Simulation Results}

In the dissertation work The multi wavelength optical orthogonal codes (MWOOC's) have been constructed and simulated by MATLAB program based on the OOC's, prime code. MWOOC's consist of 2-D codewords (or matrices) with every pulse in a codeword encoded in a distinct wavelength, supporting larger numbers of simultaneous users and subscribers without using very long code words in optical CDMA networks using multiple wavelengths, Such a characteristic is important for ensuring quality of service for real time application. For more important real-time transmission, the user will be assigned with longer signature pattern to guarantee a better quality or, in other words, error probability the Fig(2) shows three curves that used same code weight $=4$ and the number of the user in the system $\mathrm{K}=\mathrm{k} 0+\mathrm{k} 1$ where error probability will be about $10^{-3}$ and $10^{-4}$ where the probability of error is high when campared with another users using higher codeweight because increaseing the codeweight in the codeword will result in better quality of service also enhancing in the performance

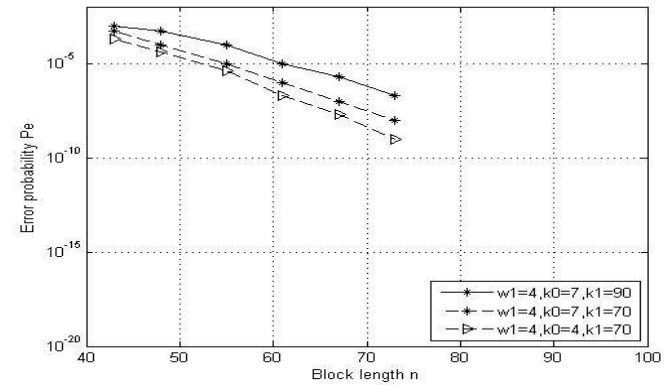

Fig(2) Variation of error probability with block length (code weight:04)

But when the code weight is increased from 4 to $7(w=7)$ we observed that the probability of error decreases to about $10^{-5}$ and $10^{-7}$ and this will give better performance as shown in the Fig(3)

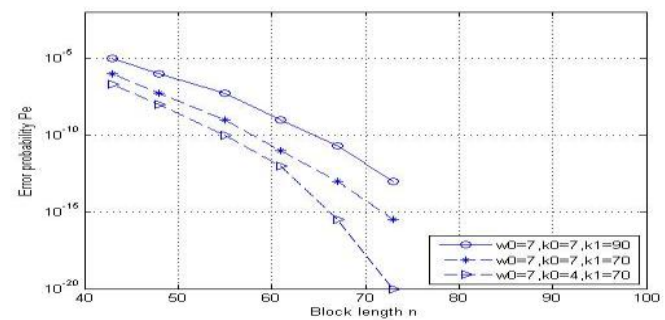

Fig(3) Variation of error probability with block length (code weight:07)

Fig(2) and Fig(3) are combined in one figure to look at the difference between two system where-in one system higher code weight for users and the after used lower code weight. Fig(4) provides important information when the number of weight increase in the codeword for the user the probability of error is decreased so as to provide better quality of service and this matches with the objective of the dissertation work. The number of weight in the codeword increases the performance would be better even when the number of users be more(traffic on the network)

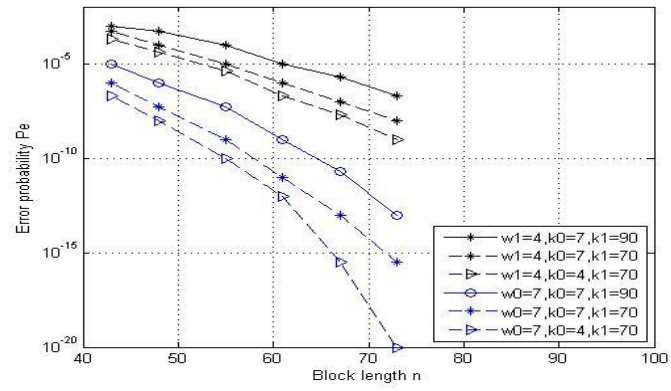

Fig(4) Camparsion of the system with in relevance to probability of error with the code weight 4 and 7 


\section{Conclusion}

In the dissertation work the error probability as a function of block length for a CDMA system with multiple weight 2D code words was investigated the simulated Multiwavelength optical orthogonal codes (MWOOC) is a more important factor determining the system performance, while the number of simultaneous users plays a lesser role . It was observed that the users having higher code weight always perform better than the users having lower code weight and this will lead to achieving guarantee for quality of service

[1]. Viterbi, A., 1979. Spread spectrum communications--Myths and realities. IEEE Commun. Mag., 17: 11-18.DOI: 10.1109/MCOM.1979.1089969

[2]. Pickholtz, R., D. Schilling and L. Milstein, 1982.Theory of spread-spectrum communications-a tutorial. IEEE Trans. Commun., 30: 855-884.10.1109/TCOM.1982.1095533

[3]. Hata, M., N. Shiraishi, S. Tomisato, 2006. Cellular CDMA system capacity in a service area with tapered traffic distribution. Proceedings of theIEEE Wireless Communications and NetworkingConference, Apr. 3-6, IEEE Xplore Press, LasVegas, NV, pp: 25-29. DOI:10.1109/WCNC.2006.1683435

[4]. Prucnal, P., M. Santoro and T. Fan, 1986. Spread spectrum fiber-optic local area network usingoptical processing. J. Lightw. Technol., 4: 547-554.DOI: 10.1109/JLT.1986.1074754

[5]. Salehi, J.A., 1989. Code division multiple-accesstechniques in optical fiber networks. I. Fundamental principles. IEEE Trans. Commun.,37: 824-833. DOI: 10.1109/26.31181

[6]. Salehi, J.A. and C.A. Brackett, 1989. Code divisionmultiple-access techniques in optical fibernetworks. II. Systems performance analysis. IEEETrans. Commun., 37: 834-842. DOI:10.1109/26.31182

[7]. Hamam, H., 2006. Optical Fiber Components: Designand Applications. 1st Edn.,Transworld ResearchNetwork, India, ISBN: 8130800977, pp: 243.

[8] M. J. Parham, C. Smythe and B. L. Weiss, "Code division multiple access techniques for use in optical fiber local area networks," Elect. AndComm. Journal, pp. 203-212, Aug. 1992.

[9] J. Salehi, "Code Division Multiple AccessTechniques in Optical Fiber Networks-Part I:Fundamental Concepts," IEEE Trans. Comm., Vol.37, p. 824, August 1989.

[10] V. Baby, B. C. Wang, L. Xu, I. Glesk and P. R.Prucnal, "Highly scalable serial-parallel opticaldelay line," Optics Communications, Vol. 218, pp.235-242, April 2003.

[11] W. C. Kwong and G.-C.Yang, "Image Transmission in Multicore-FiberCode-Division Multiple-Access Networks," IEEE Commun.Lett., vol. 2, no.10, Oct. 1998, pp. 285-87.

[12] G.-C. Yang, "Variable-Weight Optical Orthogonal Codes for CDMA Networkswith Multiple Performance Requirements," IEEE Trans. Commun., vol.44, no. 1, Jan. 1996, pp. 47-55.

[13] .Fouli, K. and M. Maier, 2007. OCDMA and OpticalCoding: Principles, Applications and Challenges.IEEE Commun. Mag., 45: $27-$ 34. DOI:10.1109/MCOM.2007.4290311

[14]. Wang, X., 2008. Recent progresses in OCDMA.Proceedings of the 10th Anniversary InternationalConference on Transparent Optical Networks, June22-26, IEEE Xplore Press, Edinburgh, pp: 39-42.DOI: 10.1109/ICTON.2008.4598365

[15] .Ashour, I.A.M., H.M.H. Shalaby and P.S. Menon,2011a. Performance analysis of SAC/opticalCDMA and WDM under a hybrid overlay scheme.Proceedings of the 2nd International Conference onPhotonics, Oct. 17-19, IEEE Xplore Press,Malaysia, pp: 1-4. DOI:10.1109/ICP.2011.6106874

[16]. Agrawal, G.P., 2002. Fiber-Optic CommunicationsSystems.3rd Ed., John Wiley and Sons Inc.

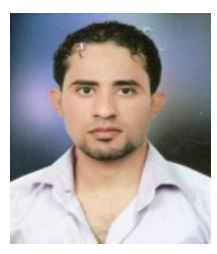

Aqeel Hamzah Sahib Al-fatlawi

Received his bachelor of Electronic and Communication Engineering Technical Najaf Collage From Iraq in 2011. and his M.Tech. in Communication System Engineering in Department of Electronics and Communication Engineering in SHIATS, Allahabad

Email:aqeelhamza88@yahoo.com From Iraq.

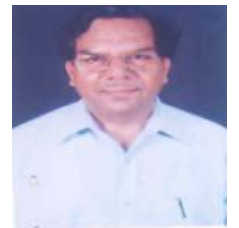

A.K. Jaiswal is Prof. and Head of ECE Dept at SHIATS-Allahabad. He Obtained M.Sc. in Tech. Electronic \& Radio Engg. from Allahabad University in1967. He guided various projects \& research at undergraduate \& postgraduate level. He has more than 35 years Industrial, research \& Teaching experience and actively involved in research and publications. His area of interest includes Optical Networks and satellite communication.

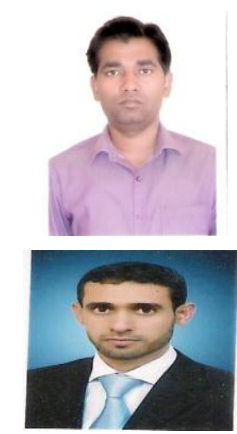

Mukesh Kumar is working as a Asst. Prof. in the Department of Electronics \& Communication Engineering in SHIATS, Allahabad

Mohanad Ahmed Abdulkareem, received his bachelor in Electronic and Communication Engineering from University of Technology in 2006 and his M.Tech.from University of Technology in 2009 from Iraq 